\title{
Exploring Oral Health Care Awareness among Intensive Care Units Nurses at KAUH
}

\author{
Jamilah S. Jastaniah1, Rawan A. Almarhoumi², Nadia A. Al-Hazmi ${ }^{3}$ \\ ${ }^{1}$ Faculty of Dentistry, King Abdulaziz University, Jeddah, Saudi Arabia \\ ${ }^{2}$ Department of Orthodontics, Faculty of Dentistry, King Abdulaziz University, Jeddah, Saudi Arabia \\ ${ }^{3}$ Oral Biology Department, Faculty of Dentistry, King Abdulaziz University, Jeddah, Saudi Arabia \\ Email: j.jastaniah@gmail.com
}

How to cite this paper: Jastaniah, J.S. Almarhoumi, R.A. and Al-Hazmi, N.A. (2017) Exploring Oral Health Care Awareness among Intensive Care Units Nurses at KAUH. Open Journal of Stomatology, 7, 488-493.

https://doi.org/10.4236/ojst.2017.711045

Received: October 10, 2017

Accepted: November 12, 2017

Published: November 16, 2017

Copyright (C) 2017 by authors and Scientific Research Publishing Inc. This work is licensed under the Creative Commons Attribution International License (CC BY 4.0).

http://creativecommons.org/licenses/by/4.0/

\begin{abstract}
The relationship between oral health status and systemic diseases is well established, however, literature shows that there is still lack of awareness among individuals taking care of ICU patients. This work aims to explore if ICU nurses are aware of the relationship between oral health status and systemic diseases, whether they practice oral health care on their patients and if they do, what methods they use. A pre-validated questionnaire was used and distributed to all nurses practicing at KAUH Medical and Surgical ICU. All surveys were voluntary and anonymous. A total of 45 of questionnaires were distributed to ICU nurses with a response rate of $95.6 \%(\mathrm{~T} n=43)$. Mean responses indicated that nurses recognize that micro-aspiration of contaminated oro-pharyngeal secretions is the most likely mechanism of transmission of bacteria into the lung of ventilated patients (mean $=5.6$ ). Accordingly, our results also showed that oral care was perceived as a very high priority for mechanically ventilated patients by $95 \%$ of nurses. Mouthwash was the primary material used for oral care by the majority of nurses $(n=32 ; 74.4 \%)$, and chlorhexidine the most used one $(\mathrm{n}=42 ; 98 \%)$. The use of manual toothbrushes was very low $(n=8 ; 23.5 \%)$ and not surprisingly, none of the nurses used electric toothbrushes to provide oral care for their ventilated patients. Finally, $86 \%$ of the respondents agreed on the need of better supplies and equipment to be provided by the hospital in order to perform better oral care in the ICU. Oral care provided in the ICU is not concordant with the global oral care protocols. Thus, the need for translation of oral care guidelines into clinical practice is of serious importance to improve the quality of care.
\end{abstract}

\section{Keywords}

Oral Health, Oral Hygiene, Nurses, ICU 


\section{Introduction}

Patients in Intensive care units (ICUs) are 24-hour monitored by nurses and depend mostly on them for their daily needs including oral care [1] [2]. It is known in the literature that poor oral hygiene compromises not only oral health in form of caries, periodontal disease but also the systemic health as bacteremia, infective endocarditis and ventilator-associated pneumonia (VAP) especially in medically compromised patients. Those mentioned diseases may worsen the health condition of ICU patients who are already vitally threatened by their primary disease [2]. Therefore, the importance of nursing personal around those ICU patients is to focus on improving the oral hygiene by removing dental plaque that provides a reservoir for microbes causing previously mentioned (systemic) diseases [3].

Most commonly as some literature shows that nurses are focusing on doing all aspects of their role and finding it more important than performing frequently oral hygiene care [4]. In contrast, a study done in Malaysia reported that $85 \%$ of ICU nurses considered oral care is a high-priority procedure for such patient [3]. Similarly, from 102 ICU unit in US $91 \%$ of nurses also showed the high importance of this oral care taking [5].

Even though there are several evidence-based oral hygiene protocols published in the literature, oral care practices still varied among not only different hospitals but also among individual nurses within the same ICU [2] [3] [4] [5]. And this difference can be related to the difficulty in removing plaque from the oral cavity as reported by $63 \%$ of American nurses [5]. When oral care is done throughout the day, majority of nurses prioritize patient comfort rather than using the effective method for plaque removal [3] [5]. For example $73.4 \%$ of more than 100 Malaysian nurses used cotton with forceps as the primary material for cleaning the oral cavity [3]. Also, it has been reported by Binkley in USA, that foam swabs and moisture agents were the primary agent used by nurses [5] rather than the recommended use of manual brushing as shown in the guideline [4]. Recently, A study done in KSA proved that the presence of oral care guideline in ICU improve the oral hygiene compared to units without guideline [1].

Another barrier to oral care is the supply provided by the hospital which undoubtedly affects the oral hygiene provided to ICU patients [2]. Sixty-nine percent of respondent in Malaysia agreed that the hospital did not even provide them with tooth brushes for their patients [3] that considered the most effective method as previously mentioned. However, ninety-six percent of respondents in US agreed that the supplies were available in the unit. But they prefer to be provided with better equipment as $46 \%$ reported [5].

Although there are a number of studies addressing the methods of oral care in ICUs worldwide, there is little information on the current practice and nurses' attitude available for Jeddah, Saudi Arabia.

The objectives of this study was to determine whether ICU nurses in Saudi Arabia practice oral health care on their patients and to assess what methods are 
used.

\section{Materials and Methods}

The questionnaire used in this study was developed by Binkley et.al. [5]. After obtaining permission from the author, this pre-validated questionnaire was distributed in its original language: English.

Ethical approval for this survey was granted by the King Abdulaziz University (KAU) the Unit of Biomedical Ethics Research Committee, Jeddah, Saudi Arabia (Reference No.50-15).

The study was a cross-sectional survey conducted at ICUs of King Abdulaziz University Hospital (KAUH) between the period of September 2014 until May 2015. The study sample was conveniently drawn from all registered ICU nurses practicing in the medical and surgical ICU (excluding pediatric ICU and Neonatal ICU nurses). The demographic data of the nurses sampled is shown in Table 1. Questionnaire were distributed by the Head ICU nurse. Participation in this survey was voluntary and anonymous. As the participant completed the questionnaire this considered as a consent form.

\section{Results}

Among 45 of total questionnaires that were distributed to ICU nurses, only 2 nurses did not fill out the questionnaire resulting in a response rate of $95.6 \%$.

The mean responses would indicate that nurses recognize that micro-aspiration of contaminated oropharyngeal secretions (mean $=5.6$ ) and preadmission colonization (mean $=5.2$ ) are the most likely mechanisms of transmission of bacteria into the lung of ventilated patients.

Oral care was perceived as a very high priority for mechanically ventilated patients by $95 \%$ of nurses. However, approximately $12 \%$ of the nurses responded that the oral cavity is a difficult body area to clean and that this procedure is unpleasant task.

Figure 1 shows the main practices for oral care carried out by the ICU nurses. Mouthwash was the primary material used for oral care by the majority of nurses every 4 hours ( $\mathrm{n}=32 ; 74.4 \%$ ), and chlorhexidine found to be the most used one ( $n=42 ; 98 \%)$. And a low percent has been recorded regarding the use

Table 1. Participants' demographic data.

\begin{tabular}{cc}
\hline Characteristics & Value \\
\hline Age (in years) - Mean - Range & $34(21-52)$ \\
ICU experience (in years) - Mean - Range & $8.5(2-26)$ \\
Educational level (number of nurses) & 34 \\
Nursing diploma program & 8 \\
Bachelor's degree & 1 \\
Missing data & \\
\hline
\end{tabular}




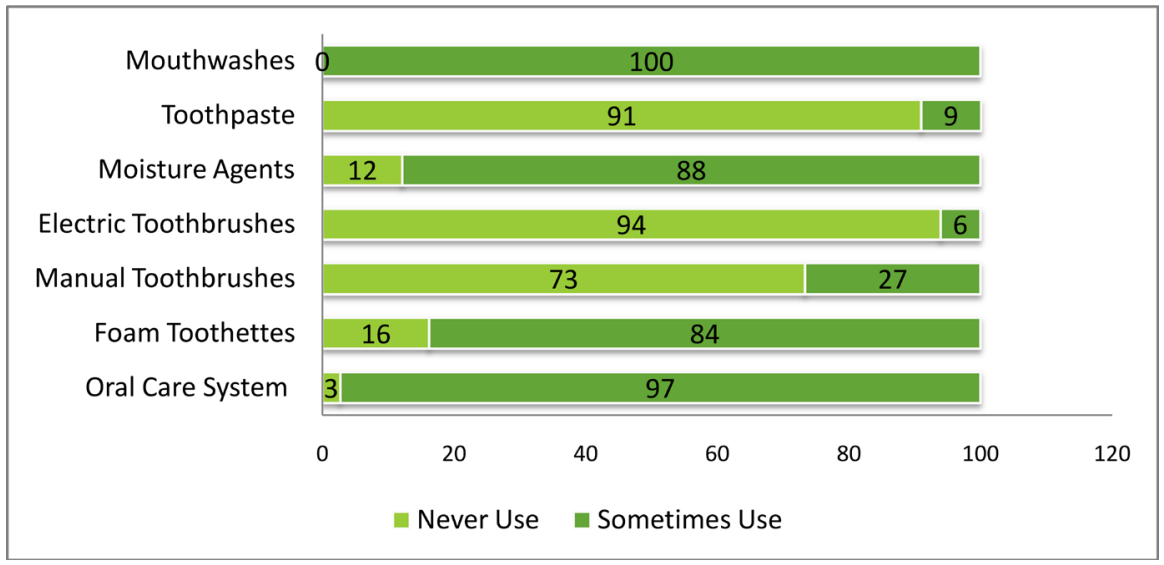

Figure 1. Types of participants' oral care practices for ICU patients.

Table 2. Nurses' attitude toward oral care practices.

\begin{tabular}{|c|c|c|c|c|}
\hline 1 & 2 & 3 & 4 & 5 \\
\hline Strongly Agree & Agree & $\begin{array}{l}\text { Neither Agree } \\
\text { nor Disagree }\end{array}$ & Disagree & $\begin{array}{l}\text { Strongly } \\
\text { Disagree }\end{array}$ \\
\hline \multicolumn{4}{|c|}{ \# Statement } & Mean \\
\hline \multicolumn{4}{|c|}{ 1) "I have adequate time to provide oral care at least once a day" } & 1.49 \\
\hline \multicolumn{4}{|c|}{ 2) "I have been given adequate training in providing oral care" } & 1.02 \\
\hline \multicolumn{4}{|c|}{ 3) "There are supplies readily available to provide oral care in our unit" } & 1.35 \\
\hline \multicolumn{4}{|c|}{$\begin{array}{l}\text { 4) "I prefer using an electric toothbrush to a manual toothbrush for cleaning } \\
\text { patients" teeth" }\end{array}$} & 3.95 \\
\hline \multicolumn{4}{|c|}{ 5) "The toothbrushes provided by the hospital are suitable for our patients" } & 2.28 \\
\hline \multicolumn{4}{|c|}{ 6) "Oral care is a very high priority for mechanically ventilated patients" } & 1.19 \\
\hline \multicolumn{4}{|c|}{ 7) "I find cleaning the oral cavity to be an unpleasant task" } & 4.44 \\
\hline \multicolumn{4}{|c|}{ 8) "The oral cavity is a difficult area of the body to clean" } & 4.37 \\
\hline \multicolumn{4}{|c|}{$\begin{array}{l}\text { 9) "No matter what I do, the mouths of most of the ventilated patients seem to } \\
\text { get worse the longer they are on the ventilator" }\end{array}$} & 3.28 \\
\hline
\end{tabular}

of manual toothbrushes $(\mathrm{n}=8 ; 23.5 \%)$.

Nurses attitudes is summarized in Table 2. In response to "I prefer that a dental hygienist perform oral care tasks?" more than $71 \%(\mathrm{n}=30)$ agreed on that and only 8 nurses disagreed. But in response to "Who do you think should be responsible for cleaning the oral cavity for intubated patients?" more than 92\% $(\mathrm{n}=38)$ responded "Nurses", $4.9 \%(\mathrm{n}=2)$ thought the dental hygienist should clean the mouth, and the reminder $(\mathrm{n}=1)$ indicated aide.

$86 \%$ of the respondents agreed regarding the need of better supplies and equipment to be provided by the hospital in order to perform a better oral care in ICU.

Finally, most of the participants $(\mathrm{n}=42$; $95 \%)$ were willing to learn more about the best way to provide oral care. Also, in response to the statement "I need more information on research-proven oral-care standards", 93\% responded 
affirmatively, and only $4.8 \%$ disagreed.

\section{Discussion}

Aspiration of contaminated secretion from oropharynx was recognizes as the most likely mechanism of transmission of bacteria into the lung and causing pneumonia which may worsen the health condition of ICU patients who are already vitally threatened by their primary disease.

Our results showed that nurses consider oral care a high priority procedure for mechanically ventilated patients (95\%) similar to other studies [3]. However, we reported a high need for better equipment in order to perform effective oral hygiene care as compared to the same study carried out by Binkley et al. (86\%, $46 \%$ respectively) [5]. Participants indicated that chlorhexidine mouthwash $0.12 \%$ was the primary material of use, and this was an encouraging finding since it is concomitant with the current best clinical guidelines for oral hygiene in the critically ill [4].

Although it is recommended to use manual brushing as a main method for plaque removal to decrease microbial colonization [5], we found that less than one-third of respondents reported the use of this method for oral hygiene care. Compared to a similar study done in Riyadh by Alotaibi et al. [1], our result suggest that more emphasis needs to be placed on this protocol. Furthermore, it could be that the majority of nurses prioritize patient comfort rather than the use of the most effective method for plaque removal as previously mentioned [3] [5]. Also, it could be due to the lack of knowledge and clinical information which could update them with research proven guidelines for oral hygiene care as mentioned to those of a study done by Ganz et al. [6].

\section{Conclusion}

Oral care provided in the ICU were not concordant with the current oral care protocols. Thus, the need for translation of oral care guidelines into clinical practice is of serious importance to improve the quality of care.

\section{Limitations}

Access to the ICU was a difficult process and there was a limited number of nurses working at KAUH which made the sample size small. Therefore, this study sheds light on KAUH ICU nurses only. Following the survey, we conducted a small workshop where we instructed the ICU nurses on the best practices for oral health care of ICU patients but due to time restraints we were unable to carry out a "post-workshop survey". In addition, because of the nature of the ICU department, we had no access to the equipment.

\section{Acknowledgements}

We would like to thank Binkley and colleagues for allowing us to use their survey. Our appreciations are also extended to Dr. Saddig D. Jastaniah for his assis- 
tant in journal submission process.

\section{References}

[1] Alotaibi, A.K., Alshayiqi, M. and Ramalingam, S. (2014) Does the Presence of Oral Care Guidelines Affect Oral Care Delivery by Intensive Care Unit Nurses? A Survey of Saudi Intensive Care Unit Nurses. American Journal of Infection Control [Internet], 42, 921-922. http://www.ncbi.nlm.nih.gov/pubmed/25087146

[2] Par, M., Badovinac, A. and Plančak, D. (2014) Oral Hygiene Is an Important Factor for Prevention of Ventilator-Associated Pneumonia. Acta Clinica Croatica, 53, 72-78.

[3] Soh, K.L., Ghazali, S.S., Soh, K.G., Abdul, R., Sharifah, R., Abdullah, S.S., et al. (2012) Original Articles Oral Care Practice for the Ventilated Patients in Intensive Care Units: A Pilot Survey. The Journal of Infection in Developing Countries, 6, 333-339

[4] Berry, A., Davidson, P., Nicholson, L., Pasqualotto, C. and Rolls, K. (2011) Consensus Based Clinical Guideline for Oral Hygiene in the Critically Ill. Intensive and Critical Care Nursing [Internet], 27, 180-185.

http://search.ebscohost.com/login.aspx?direct=true\&db=c8h\&AN=2011196544\&sit e=ehost-live

[5] Binkley, C., Furr, L.A., Carrico, R. and McCurren, C. (2004) Survey of Oral Care Practices in US Intensive Care Units. American Journal of Infection Control [Internet], 32, 161-169. http://www.ncbi.nlm.nih.gov/pubmed/15153928

[6] Ganz, F.D., Ofra, R., Khalaila, R., Levy, H., Arad, D., Kolpak, O., et al. (2013) Translation of Oral Care Practice Guidelines into Clinical Practice by Intensive Care Unit Nurses. Journal of Nursing Scholarship [Internet], 45, 355-362.

http://www.ncbi.nlm.nih.gov/pubmed/23731065 